\title{
The Role of Lateral Crural Turnover Flaps in Enhancing the Supra-alar Crease in Middle Eastern Noses
}

\author{
RIHAM LASHIN, M.D. and AHMED EL-SHAHAT, M.D. \\ The Department of Plastic Surgery, Faculty of Medicine, Ain Shams University
}

\begin{abstract}
Background: Middle Eastern noses are characterized by thick sebaceous skin and week alar cartilage, which may leads to poorly defined nasal tip, depressed tip, poorly defined ala, ill-defined supra-alar crease, and external nasal valve collapse.

Aim of Work: Is to investigate the lateral crural turnover flap technique as a method to enhance the supra-alar crease in those patients, by strengthening the lateral crura of the week lower lateral cartilage.

Material and Methods: The study included 20 Middle Eastern patients, 14 females and 6 males, Age ranging from 25-45 years, all patients underwent lateral crural turnover flaps while doing their open rhinoplasties. We used this flap in our study to strengthen the lateral crura of the week lower lateral cartilage in Middle Eastern noses, and at the same time to gain the advantage of having the same effect of doing cephalic trim in these noses, but without excising the cartilage. This turnover flap results in tip definition, tip elevation, and it gives a well-defined supra-alar crease which results in a more alar definition and leads to a more narrowing of the caudal part of the nose.
\end{abstract}

Results: All of the study patients were satisfied with the aesthetic appearance of their noses postoperative.

Conclusion: The turnover alar cartilage flap improves the esthetic appearance of the nasal tip by enhancing the the alar and supra-alar crease definition of Middle Eastern noses.

Key Words: Alar cartilage - Lateral crus - Turnover flap Supra-alar crease.

\section{INTRODUCTION}

Middle Eastern noses are characterized by thick sebaceous skin and week alar cartilage, which may leads to poorly defined nasal tip, depressed tip, and external nasal valve collapse. Weak alar definition and absent supra- alar creases which are common problem in Middle Eastern noses are usually the result of excessive soft tissue at the nasal tip or, more commonly, cartilaginous enlargement and excessive arching of the lower lateral cartilages (LLC), causing convexity [1].
Correction of poor alar definition of MiddleEastern noses has been typically achieved through a variety of cartilage-modifying manoeuvres based on incision or excision of cartilaginous structures, generally the lateral crura (LC) and the middle crura (MC) of the LLC [2]. Many surgeons routinely resect the cephalic portion of the LC of the LLC known as cephalic trim to correct the poorly defined alar deformity [3]. After the cephalic portion of LLC has been resected, there are frequent deformities that may happen due to the resultant weakening of the LLC, like external valve dysfunction, alar retraction, alar pinch, tip asymmetry, and lack of tip definition [4].

Lateral crural turnover flap technique instead of excising the cephalic part of the LL of LLC have been previously described by Janis et al., in 2009. This technique was mainly described to strengthen the weak alar cartilage to improve the external nasal valve function and prevent its collapse and to correct the lower lateral crural deformities [5]. This flap technique have the advantage of strengthening the weak alar cartilage, in addition to avoidance of the drawbacks of doing cephalic trim technique [4].

Failure to create the supra-alar creases or groove will worsen the nasal shape after performing the osteotomies in rhinoplasties, as after doing the osteotomies, the cephalic part of the nose will be too narrow, while the caudal part will appear too wide. Previous trial by Elminawi et al., in 2012 were done to create this supra-alar crease by using trancutaneous suturing. By this suturing technique, supra-alar creases were created, which not only narrows but also increases tip projection, but it results in evident and unnatural skin depression [6]. 
Another previous study was done by Loganathan Selvaraj in 2016, who achieved constriction of the upper part of alar sidewall and deepening of supra-alar creases by supra- alar cinching through an intraoral approach [7]. Unlike the transcutaneous suturing technique described by Elminawi et al., in 2012 [6], scar formation in the skin was totally avoided with Selvaraj procedure [7].

In the current study, we used the lateral crural turnover flap technique to improve the aesthetics of Middle Eastern noses. As in our current study, we used the lateral crural turnover flap instead of doing cephalic trim of lateral crura of lower lateral cartilage to avoid weakening the lateral crura of the already week lower lateral cartilage which happens with the cephalic trim technique, and at the same time to gain the advantage of having the same effect of doing cephalic trim in these noses, but without excising the cartilage. We used this technique also to create a more natural supra-alar creases without transcutaneous suturing which appear evident and unnatural.

\section{Aim of work:}

The aim of this study is to investigate the lateral crural turnover flap technique as a method to enhance the alar definition and to enhance the aesthetically favored supra-alar creases in Middle Eastern noses by strengthening the lateral crura of the week lower lateral cartilage.

\section{MATERIAL AND METHODS}

In the present study, 20 primary rhinoplasties using lateral crural turnover flaps were included. The study were performed between January 2016 and September 2017. There were 14 females $(70 \%)$ and 6 males (30\%), age of patients ranging from 25-45 years with mean age of 32.6 years as shown in Table (1). The mean follow-up period was 12 months. Photography was done for all patients as part of a pre-operative and postoperative clinical assessment for an exact evaluation of the results.

An evaluation of the results was done using pre and post-operative photographs (1 week, 3, 6, and 12 months after the surgery), and a patients' satisfaction poll. Patients' satisfaction was classified from 1 (not satisfied) 2 (acceptable) 3 (good) 4 (very good) to 5 (completely satisfied) [6]. Patients were selected based on their preoperative standard photographs, preoperative examination, and intraoperative finding.
The exposure provided by the open approach allowed direct assessment of tip cartilages in natural and undistorted positions. The LC were horizontally demarcated, preserving at least $5-6 \mathrm{~mm}$ of the alar cartilage caudally.

The indications for lateral crural turnover flap technique were (beside their wish to reduce the size of nasal tip that was too wide or too bulbous) as follows: The lateral crura should be strong enough, not less than $10 \mathrm{~mm}$ wide, convex or straight, patient's skin should be normal or thick not to show the flap.

The exclusion criteria in our study were: Alar pinching, the width of lateral cruras is less than $10 \mathrm{~mm}$, weak lateral crura and thin skin; because the flap technique was not applicable in those cases. The process was explained to all patients, who signed an informed consent afterward.

\section{Operative technique:}

All patients underwent open rhinoplasty by the senior author (Elshahat A.), in which the lower lateral crural turnover flap was used with or without other grafting techniques, additionally to other rhinoplasty components, such as dorsal hump reduction, osteotomies, septoplasty, tip modifying techniques, and so on.

After intubation and sterile preparation were done. Local injection of epinephrine 1:100,000 was injected in a standard fashion. Before making the initial incision, we wait for the mandatory time period till the adrenalin effective.

The lower lateral crura are approached before any tip shaping maneuvers are performed. Standard stepladder incision was made across the columella using a number 15 blade. Sharp curved scissors were used to make bilateral infra-cartilagenous incisions $2 \mathrm{~mm}$ back from nostril rim, which were then connected to the marginal extension of the columellar incision.

Sharp dissection with a scissor was made; it preserved the subdermal plexus to the delicate skin of the columella, tip, and soft tissue triangles.

Identification of the LLCs was followed by exposure of the cartilaginous dorsum. A periosteal elevator was used to dissect the periosteum from the nasal bones in an extended way. Bilateral mucoperichondrial septal flaps were elevated exposing the septal cartilage. Then septoplasty with lateral retraction of the LLCs and identification of 
the anterior septal angle was done. For avoiding destabilization of the keystone area, the harvesting of septal cartilage was postponed until the work on the upper third of the nose has been completed. Next, the bony dorsal contour and the middle vault were managed, based on the desired final tip position. If required, dorsal refinements and osteotomies were done. After septoplasty, spreader grafting, etc., the tip plasty began.

We marked the turning line along the lateral crura at the superior junction of the lower lateral cartilage and the accessory cartilage complex through to the lateral domal margin. A 4- to-5mm total width of lower lateral cartilage is turned over depending on the inherent total width, thickness, and deformity of the cartilage. The average width of the native lower lateral cartilage should therefore be greater than $1 \mathrm{~cm}$ to accommodate a lower lateral crural turnover flap to preserve at least a $6-\mathrm{mm}$ caudal rim strip.

For repositioning of the lateral crura. The vestibular skin is undermined off the entire cephalic border of the cartilage by blunt dissection until the turning point of the flap are reached on the undersurface of the cartilage. We kept remaining lateral crura attached to the vestibular skin to preserve its natural stability. The dissection was done on the plane keeping perichondrium attached to the cartilage on both sides. Once the lateral cruras of the LLCs were partially free from skin, not a complete cut, but a slight incision (scoring) by 15 blade scalpel under the lateral cruras was done according to the previously drawn line, the integrity of the anterior surface of the cartilage and its perichondrium were preserved. While, a 1 to $2 \mathrm{~mm}$ complete cut incision was made to decrease the tension at the medial and lateral ends of the cartilage. The cephalic cartilage flap is then turned over onto the remnant caudal cartilage as a flap, by this turning, the convex cartilage was reshaped by its tension, and the lateral cruras were straightened. The contra force of two convex turned over parts of LLC makes it straight and strong by itself.

By using Adson Brown forceps to stabilize and flatten the cartilage, the turnover flaps are secured to each other with a series of three to four caudally based horizontal mattress 5-0 Proline sutures. Uncut vessels give blood supply to the flap that makes it more capable of surviving and providing the support to remaining lateral crura. Care is taken to avoid incorporating the vestibular skin in the cartilaginous reapproximation.
After alar flap technique was performed, the surgeon was checking the sufficiency of the lateral wall and evaluated for adequate correction of the anatomic defect and for improved alar support. The two-layer lower lateral cartilages will now appear flat with a gentle cephalic orientation.

This technique is employed before performing tip suturing. After that, we managed the projection and rotation of tip, if it was needed. Columellar strut graft was placed, and medial crura of the LLCs were fixed to it in all patients [8]. Then interdomal and transdomal sutures were applied as needed. After everything was done with the nose skeleton, we redraped the skin envelope, evaluating if additional grafts were needed or not. The closure was begun with a single subcutaneous 5-0 Vicryle suture in the midline of the collumelar incision for more symmetrical matching of skin envelope edges. Simple interrupted 7-0 Proline sutures were used to close and slightly evert the stepladder collumelar incision. The infra-cartilagenous incisions were closed with 5-0 Vicryle sutures in a simple interrupted fashion watching the nostril margin closely. The septum was closed by reapproximating the mucoperichondrial flaps with a 3-0 Rapid Vicryle plain trans-septal suture in a running mattress fashion. The nose was then taped, splinted, and a cast was applied in a routine fashion.

\section{RESULTS}

The current study include 20 Middle Eastern patients, 14 females (70\%) and 6 males (30\%), Age ranging from 25-45 years with mean age of 32.6 years as shown in Table (1). The lower lateral crural turnover flap technique was performed during 20 primary rhinoplasties performed upon the study patients.

We measured the percentage of the supra-alar width divided by the lip width to standardize our measurements, and we compared this percentage pre and postoperative for each nose, and we produced the decrease in the percentage of the supraalar width postoperatively for each of the 20 patients (Fig. 1). The percentage of the preoperative supra-alar width divided by the lip width ranging from $41.2 \%-65.5 \%$, with mean percentage of $54.1 \%$. While percentage of the postoperative supra-alar width divided by the lip width ranging from $30.8 \%-43.3 \%$, with mean percentage of $34.3 \%$. The percentage of the decrease in the postoperative supra-alar width ranging from $6.2 \%$ $34.5 \%$, with mean decrease of $19.7 \%$. 
Statistical analysis was done showing that a lower mean percentage of the supra-alar width divided by the lip width postoperative is $34.3 \%$, compared to $54.1 \%$ preoperative, with the difference is highly significant statistically using the paired $t$-test. With a mean decrease of $-19.7 \%$ after surgery among the studied patients as shown in Table (2). Based on the mean preoperative width, the postoperative decrease in width is $(36.6 \%)$ of the preoperative width.

Primary rhinoplasty patients usually have adequate available lower lateral crura; therefore, this technique was used in primary cases. No cases of alar notching, buckling at the corners of the ala, or external valve collapse were seen postoperatively. The long-term results of the flaps were excellent, with all patients showing contour retention and lasting results in their 6-month and 1-year postoperative photographs. The revision rate and asymmetry in this study was zero percent. There were no complications such as hematoma, suture infection or reaction, suture visualisation, or a need to remove the sutures.

This underscores the importance of incising the full thickness of the cartilage at the medial and lateral margins of the lower lateral crural turnover flap and the need to completely score the entire length on the posterior lower lateral crura, ensuring that there is minimal to no tension on the corners as the flap is being turned over and sutured down. Buckling that may be seen at the corners is corrected by excision, additional suture placement, or further scoring, but it doesn't happen with any of our patients. It happens more commonly in patients with a thin skin envelope, as otherwise visible deformities can result.

Patient satisfaction was measured as regard the aesthetic appearance of the nose, using a numeric survey from 1-5, with number 1 indicates poor (not satisfied) results, number 2 indicates fair (acceptable) results, number 3 indicates good results, number 4 indicates very good, and number 5 indicates excellent (completely satisfied) results [6]. Satisfactory results were achieved. All of the study patients were satisfied with the aesthetic appearance of their noses postoperative. All patients had harmonious aesthetic tip contouring with well defined supra-alar creases after the surgery (Figs. 2-9). The average patient' satisfaction was 4 in a scale from 1-5.

Statistical analysis for the patient satisfaction was analyzed showing that $5 \%$ (1 patient) reported good results, $40 \%$ (8 patients) reported very good results, and 55\% (11 patient) reported excellent results and were completely satisfied aesthetically as shown in Table (3).

Comparing the satisfaction level of the aesthetic appearance of nose postoperative to the mean reduction in alar width postoperative was also analyzed statistically, showing that the patients who reported good and very good satisfaction level (9 patients), have mean reduction of $-19.8 \%$ of their alar width postoperative, while the patients who reported excellent satisfaction level (11 patients), have mean reduction of $-19.6 \%$ of their alar width postoperative. But no significant difference statistically between good and excellent level of satisfaction and the mean reduction of alar width postoperative using the paired $t$-test as shown in Table (4).

Table (1): Demographic data of studied patients.

\begin{tabular}{llll}
\hline Gender & No. & $\%$ & \\
\hline Male & 6 & 30.0 & \\
Female & 14 & 70.0 & \\
\hline Age & Mean & SD & Range \\
& 32.6 & 6.0 & $25-45$ \\
\hline
\end{tabular}

Table (2): Comparison between preoperative and postoperative percentage of supra-alar width divided by the lip width.

\begin{tabular}{lcccc}
\hline & Mean & SD & Paired $t$ & $p$ \\
\hline Pre-operative & 54.1 & 6.8 & & \\
Post-operative & 34.3 & 3.4 & 11.9 & $0.000^{* *}$ \\
\hline
\end{tabular}

$* * p<0.01$ highly significant.

Table (3): Distribution of patient satisfaction.

\begin{tabular}{lll}
\hline $\begin{array}{l}\text { Aesthetic appearance } \\
\text { of nose }\end{array}$ & No. & $\%$ \\
\hline Good & 1 & 5.0 \\
Very good & 8 & 40.0 \\
Excellent & 11 & 55.0 \\
\hline
\end{tabular}

Table (4): Comparison between the level of satisfaction of the aesthetic appearance of nose and the percentage of the mean reduction of alar width postoperative.

\begin{tabular}{lcccc}
\hline Level of satisfaction & $\begin{array}{c}\text { Mean } \\
\text { Reduction } \%\end{array}$ & SD & $t$ & $p$ \\
\hline $\begin{array}{l}\text { Good or very good } \\
\mathrm{N}=9\end{array}$ & -19.8 & 6.9 & 0.07 & 0.9 \\
$\begin{array}{l}\text { Excellent } \\
\mathrm{N}=11\end{array}$ & -19.6 & 8.1 & & \\
\hline
\end{tabular}

$p>0.05$ not significant. 

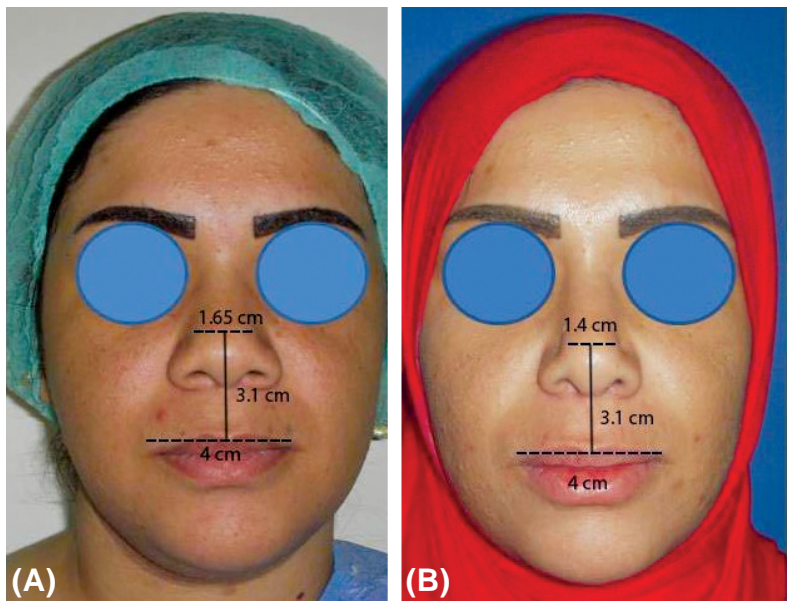

Fig. (1): A photo showing measuring the percentage of the supra-alar width divided by the lip width to standardize our measurements, then comparing this percentage pre (A) and postoperative (B) for a 34 years old, female patients with broad dorsum of nose, poorly defined tip and ala of nose, wide nasal ala, and ill-defined supra-alar creases. Postoperative photo show narrower nasal dorsum, well defined elevated nasal tip, and aesthetically pleasant supra-alar creases.
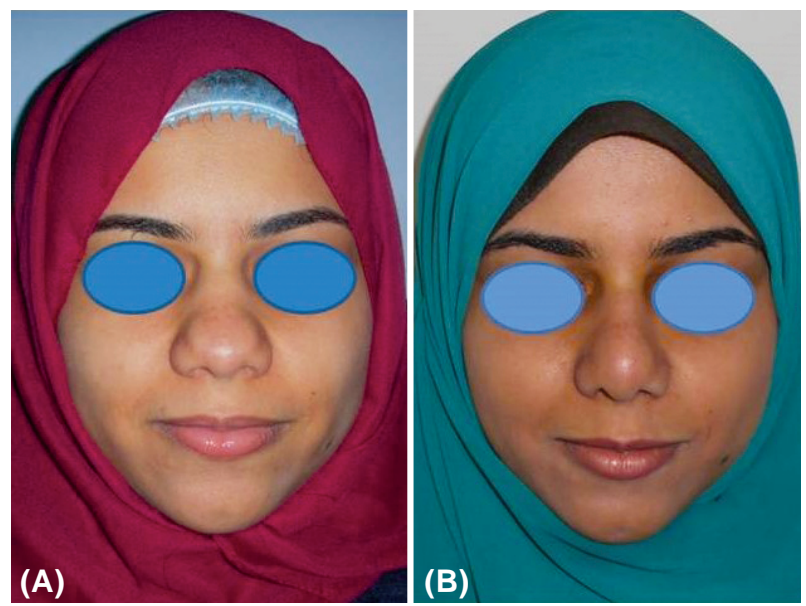

Fig. (3): A photo showing 26 years old, female patients with broad dorsum of nose, depressed nasal dorsum, bulbous tip, poorly defined ala of nose, and ill-defined supra-alar creases. (A) Showing preoperative photo. (B) Show postoperative photo for the same patient with narrower elevated nasal dorsum, well defined elevated nasal tip, and aesthetically pleasant supra-alar creases.
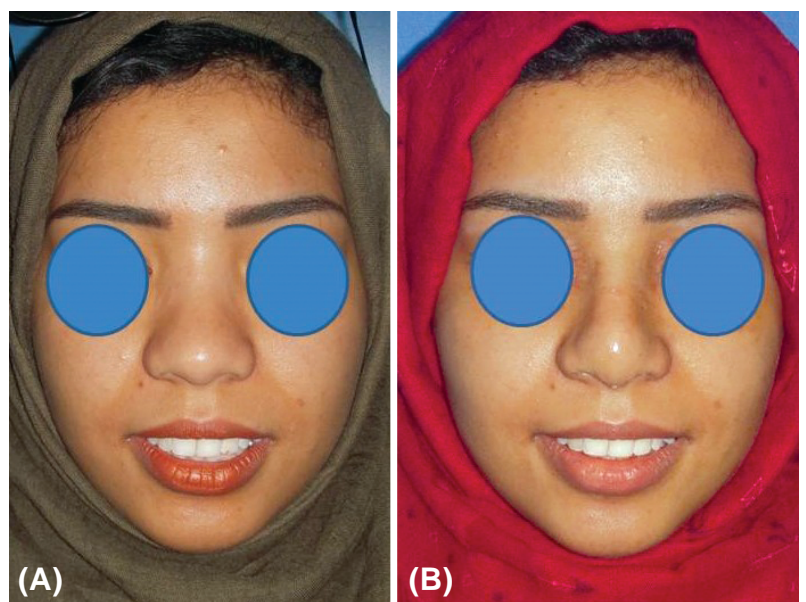
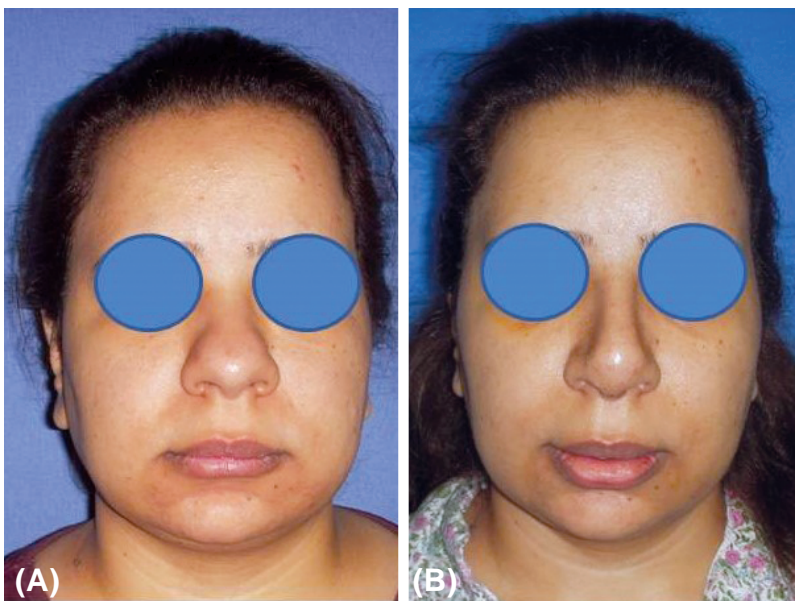

Fig. (2): A photo showing 32 years old, female patients with broad dorsum of nose, bulbous tip, poorly defined ala of nose, and ill-defined supra-alar creases. (A) Showing preoperative photo. (B) Show postoperative photo for the same patient with narrower nasal dorsum, well defined elevated nasal tip, and aesthetically pleasant supra-alar creases.
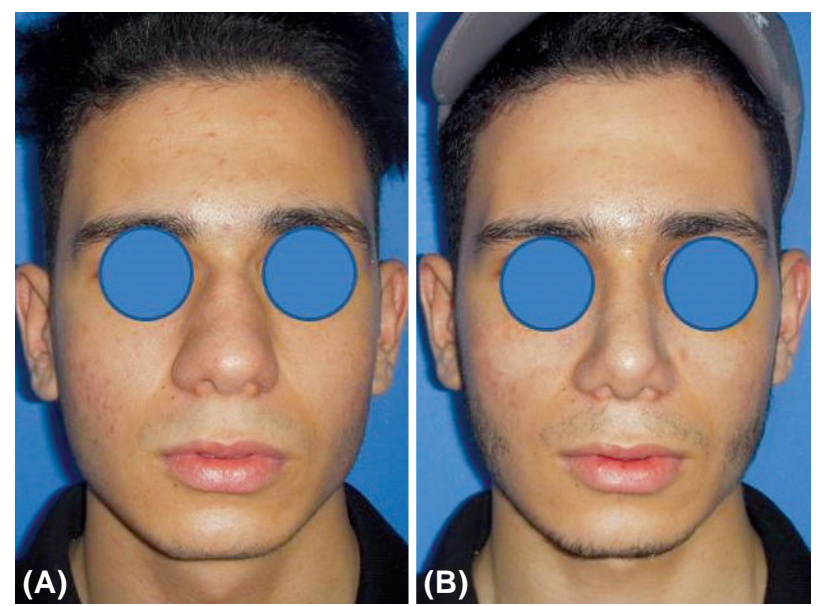

Fig. (4): A photo showing 28 years old, male patients with broad dorsum of nose, deviated nose, bulbous tip, poorly defined ala of nose, and ill-defined supra-alar creases. (A) Showing preoperative photo. (B) Show postoperative photo for the same patient with narrower centralized nasal dorsum, well defined nasal tip, and aesthetically pleasant supra-alar creases.

Fig. (5): A photo showing 25 years old, female patients with broad depressed dorsum of nose, bulbous tip, poorly defined ala of nose, and ill-defined supra-alar creases. (A) Showing preoperative photo. (B) Show postoperative photo for the same patient with narrower elevated nasal dorsum, well defined elevated nasal tip, and aesthetically pleasant supra-alar creases. 


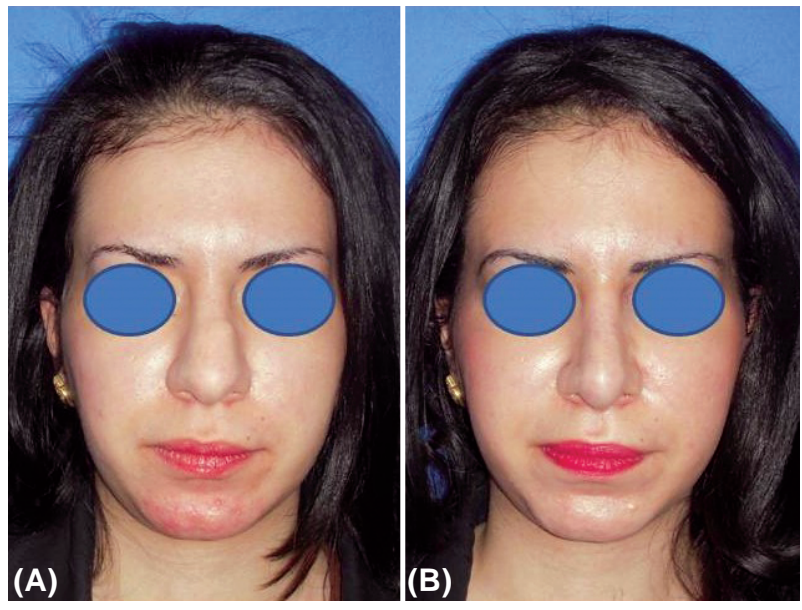

Fig. (6): A photo showing 30 years old, female patients with broad deviated dorsum of nose, bulbous tip, poorly defined ala of nose, and ill-defined supra-alar creases. (A) Showing preoperative photo. (B) Show postoperative photo for the same patient with narrower centralized nasal dorsum, well defined elevated nasal tip, and aesthetically pleasant supra-alar creases.

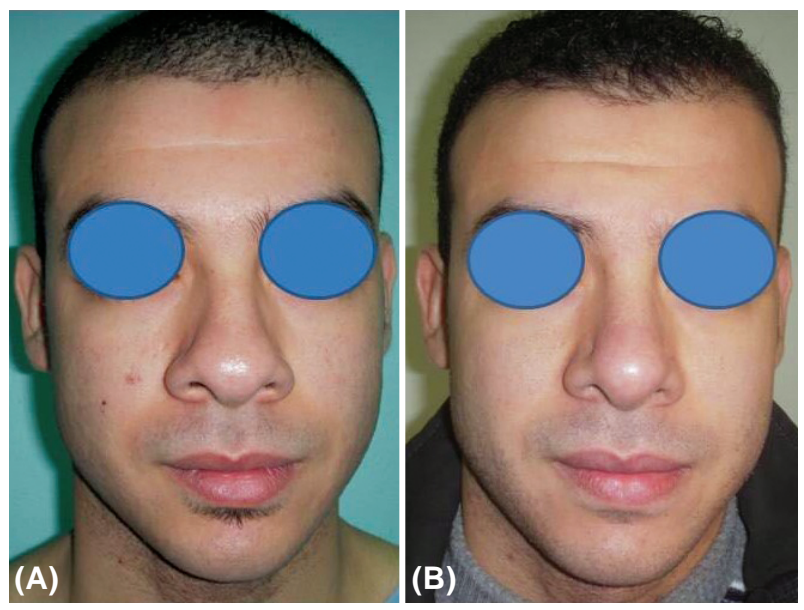

Fig. (8): A photo showing 31 years old, male patients with bulbous tip, depressed tip, wide ala, poorly defined ala, and ill-defined supra-alar creases. (A) Showing preoperative photo. (B) Show postoperative photo for the same patient with well defined elevated nasal tip, and aesthetically pleasant supraalar creases.

\section{DISCUSSION}

The Middle Eastern noses with its thick skin, and weak alar cartilage, results in an ill-defined supra-alar crease and poor alar definition in those patients. Failure to create this supra-alar crease will worsen the nasal shape after performing the osteotomies in rhinoplasties, as after doing the osteotomies, the cephalic part of the nose will be too narrow, while the caudal part will appear too wide. Previous techniques were prescribed to enhance this supra-alar crease or groove, among these techniques, there was a study by Elminawi et al., in 2012 were done to create this supra-alar crease

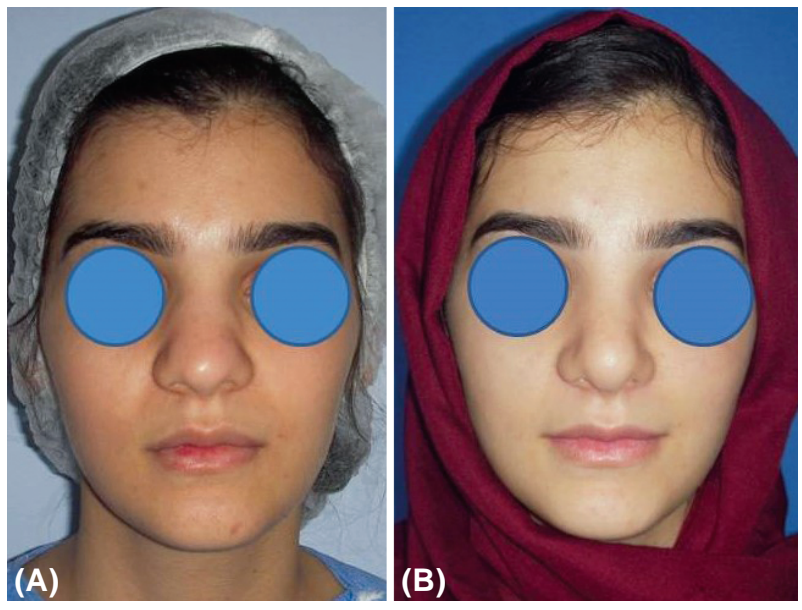

Fig. (7): A photo showing 27 years old, female patients with broad depressed dorsum of nose, deviated nose, bulbous tip, poorly defined ala of nose, and ill-defined supra-alar creases. (A) Showing preoperative photo. (B) Show postoperative photo for the same patient with narrower centralized nasal dorsum, well defined nasal tip, and aesthetically pleasant supra-alar creases.

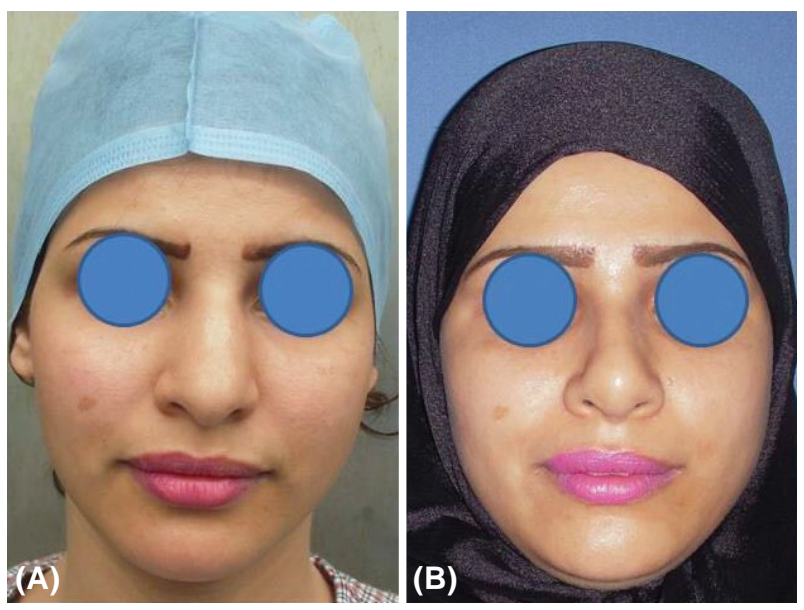

Fig. (9): A photo showing 29 years old, female patients with broad dorsum of nose, deviated nose, bulbous depressed tip, hanging collumella, poorly defined ala of nose, and illdefined supra-alar creases. (A) Showing preoperative photo. (B) Show postoperative photo for the same patient with narrower centralized nasal dorsum, well defined elevated nasal tip, and aesthetically pleasant supra-alar creases.

by using transdomal suturing, with resection of part of the cephalic plate of lower lateral cartilage together with transcutaneous lateral crural spanning sutures. Then fixing the domal cartilage to the overlying skin [6]. By this suturing technique, supra-alar creases were created, which not only narrows but also increases tip projection, but it results in evident and unnatural supra-alar creases.

Another previous study was done by Loganathan Selvaraj in 2016, who achieved constriction of the upper part of alar sidewall and deepening of supra-alar creases by simple supra-alar cinching through an intraoral approach in patients with 
widened supra-alar sidewall and ill-defined supraalar crease. Intraoral maxillary vestibular incision was done and the lateral part of the supra-alar tissue was anchored to the adjacent bone (near the pyriform rim) resulted in constriction of supra-alar region and attenuation of the alar crease. The advantages of this approach were easy accessibility, visibility, and lesser incidence of complications [7]. Unlike the transcutaneous suturing technique described by Elminawi et al., in 2012, scar formation in the skin was totally avoided with Selvaraj procedure.

Enhancing the Middle Eastern nose aesthetics in the current study depends on deepening the supra-alar creases, which will provide alar constriction of the upper part of alar sidewall creating the harmony between the upper part of the nose which appear narrow after performing the osteotomies and the lower part. In the current study we created this supra-alar groove or crease using the lateral crural turnover cartilage flap with natural definition of ala. Suturing techniques either the transcutaneous sutures [6] or the intra-oral cinching sutures [7] may not be as strong, durable, or predictable as the creation of this bilaminate lower lateral crural construct used in our study [9-14].

The advantages of the lateral crural turnover flap technique are: Utilization of adjacent expendable cartilage with preservation of its inherent vascularity, it does not need any additional grafting; it is quick and safe to perform; it has stable results. Some limitations to this technique are found, as it needs a wide alar cartilage, as assurance that there is adequate cephalic lower lateral crural cartilage is mandatory, so we can leave at least $6 \mathrm{~mm}$ width of caudal lateral crural cartilage after transposing and turning over the flap. It is contraindicated when there is insufficient width of the lower lateral crura [5].

One of the most complicated aspects in rhinoplasty is resolving and preventing functional compromise of the nasal valve area consistently. In Middle Eastern noses, the lateral crura of the lower lateral cartilage are convex or dome shaped, and its dimensions are enlarged. This cartilaginous misshapenness, although aesthetically undesirable, is functional because it keeps the nostrils open and provides good support for the nasal tip. Aesthetic rhinoplasty may cause or worsen airway obstruction in bulbous tip deformities [15].

Various techniques for tip reshaping, such as cartilage reduction techniques and alar cartilage suturing, may narrow the bottleneck of the nostrils and deteriorate the external valve. Resection of significant portions of the lower lateral cartilages during rhinoplasty or postoperative soft tissue contraction can destroy external valvular support and impair the airway [16].

Conservatively reducing the volume of the cephalic portion of the LC and preserving the greater part of the crus while maintaining a complete (uninterrupted) strip of LLC are ideal. Therefore, lateral crural turnover flap can be used to avoid external nasal valve dysfunction, in addition, it provides aesthetic nasal tip improvements and increases a visual gain in tip position without functional impairments as successfully as cephalic excision $[\mathbf{1 7 , 1 8}]$.

Numerous previous techniques were suggested to correct weak alar cartilage: The lateral crural strut graft (Gunter's graft) is widely and successfully used from the time it was found till now; however, it has some unfavorable outcomes, such as visible or palpable grafts and others. Also in some patients, there is lack of septal cartilage for using as the graft material, so it becomes necessary to harvest it from other donor sites, such as ear or rib cartilage [19]. The lateral crural turnover flap technique used in the current study does not require an extra cartilage graft material. It also can be used safely in cases with lack of septal cartilage for any reason, in addition that with this technique none of the previous deformities happened, such as visible or palpable grafts and others.

McCollough and Fedok, in 1993 described a simple technique to correct lower lateral crural concavities using the "lateral crural turnover graft". Their technique has the advantage of augmenting the lower lateral crura with native, adjacent tissue that would otherwise be removed as a cephalic cartilage remnant, thereby obviating the need for harvest from a different cartilage site [20].

Janis et al., 2009 modified McCollough's lower lateral crural turnover graft (McCollough's and Fedok, 1993) and creating a vascularized lower lateral crural flap that is connected by intact anterior perichondrium, turned over, and sutured to the remaining caudal lower lateral crural segment, instead of excising this cephalic part [5].

Conventional cephalic excision of the lateral crura can exaggerate both functional and aesthetic problems in the management of weak LLC [3]. As after the cephalic portion of LLC has been resected in the cephalic trim technique, there are frequent deformities that may happen, like external valve dysfunction, alar retraction, alar pinch, tip asym- 
metry, and lack of tip definition [21]. None of these postoperative alar deformities happened with lateral crural turnover flaps.

Janis et al., in 2009 who described the lateral crural turnover flap technique in 24 patients, with fellow-up time of 1 year, found that this technique is a versatile and reproducible technique for correction of lower lateral crural deformities and improvement of external valve function, it has been applied to the correction of lower lateral crural concavities, weakness, collapse of the lower lateral crura, strengthening the external valve, and prevention of lower lateral crural weakness from tip suturing effects [5].

The biomechanical benefit to this technique stems from the fact that the concave cephalic segment is transposed over the caudal segment and, in doing so, creates a convex-to-concave interposition of opposing intrinsic forces, which serves to strengthen and support the remaining lower lateral crural segment and support the alar $\operatorname{arch}[5]$.

Many modifications to the lateral crural turnover flaps were previously described, as Goksel and Vladykina, in 2017 introduced the oblique turnover flap technique (OTFT) for repositioning and flattening of the lateral crura of LLC in their study which included 24 patients aging from 23-46 years old, with fellow-up time of 12 months by turning the cephalic part of the lateral cruras over the caudal part in an oblique way as a flap, which leads to repositioning and flattening of the lateral crura at the same time, without using grafts. The mean satisfactory ratings of nasal tip appearance and breathing quality were significantly improved. They concluded that OTOF is a quick, useful, efficient technique for repositioning and flattening of the lateral cruras of CMLLC with a good, predictable, and stable long-term results [22].

Another modification was described by Suzgar in 2010, who described horizontal reduction with a cephalic hinged flap of the lateral crura $(\mathrm{HRCH})$ in a study included 28 patients who underwent open rhinoplasty for management of the bulbous tip. Satisfactory results were achieved at 14 months postoperative fellow-up period. Our criticism to this technique that when doing the double scoring of the cephalic part of LLC and turning it as a hinged flap, this turning point will be a weak point, and the cephalic cartilage will survive as a graft rather than a flap. While in the lateral crural turnover flap technique used in our study, the turning point of the lateral crura being double layered is considered a strength point to the lateral crura [1].
Previous studies like Janis et al., 2009 [5] and Goksel and Vladykina, in 2017 [22] used 5-0 Vicryl sutures to stabilize the lateral crural turnover flap, while in our study we used 5-0 proline sutures to stabilize the flap to maintain long lasting results with non-absorbable sutures, in addition to lower incidence of infection.

Rhinoplasty is a complex, comprehensive operation that needs a lot of techniques and maneuvers to reach the goal of aesthetic and functional satisfaction.

\section{Conclusion:}

The lower lateral crural turnover flap is a useful and reproducible technique in open rhinoplasty with enduring results. The use of adjacent cartilage provides a local source of viable tissue to correct and support the lower lateral crura in primary rhinoplasty.

As a result of our study, we can conclude that lateral crural turnover flap is a useful, quick, and safe technique for surgical treatment of Middle Eastern noses with ill-defined ala. The turnover alar cartilage flap improves the esthetic appearance of the nose by enhancing the the alar and supraalar crease definition of Middle Eastern noses.

\section{REFERENCES}

1- Suzgar A.: Horizontal Reduction Using a Cephalic Hinged Flap of the Lateral Crura: A Method to Treat the Bulbous Nasal Tip. Aesthetic Plastic Surgery, 34 (5): 642, 2010.

2- McKinney P.: Management of the bulbous nose. Plast. Reconstr. Surg., 106: 906, 2002.

3- Nagarkar P1, Stark R.Y., Pezeshk R.A., Amirlak B. and Rohrich R.J.: Role of the Cephalic Trim in Modern Rhinoplasty. Plast. Reconstr. Surg., 137 (1): 89, 2016.

4- Gubisch W. and Eichhorn-Sens J.: Overresection of the lower lateral cartilages: A common conceptual mistake with functional and aesthetic consequences. Aesthetic Plast. Surg., 33: 6, 2009.

5- Janis J.E., Trussler A., Ghavami A., Marin V., Rohrich R.J. and Gunter J.P.: Lower Lateral Crural Turnover Flap in Open Rhinoplasty. Plast. Reconstr. Surg., 123 (6): 1830, 2009.

6- El Minawi H.M., Sobhi A.M. and Magdi F.: Rhinoplasty for the Thick Skin. Egypt, J. Plast. Reconstr. Surg., 36 (1): 5, 2012.

7- Selvaraj L.: Novel technique and simple approach for supra-alar region and supra-alar crease correction by supra-alar cinching. Natl. J. Maxillofac. Surg., 7 (1): 108, 2016.

8- Rohrich R.J., Hoxworth R.E. and Kurkjian T.J.: The role of the columellar strut in rhinoplasty: Indications and rationale. Plast. Reconstr. Surg., 129 (01): 118, 2012. 
9- Tebbetts J.B.: Shaping and positioning the nasal tip without structural disruption: A new systematic approach. Plast. Reconstr. Surg., 94: 61, 1994.

10- Neu B.R.: Suture correction of nasal tip cartilage concavities. Plast. Reconstr. Surg., 98: 971, 1996.

11- Gruber R.P., Park E., Newman J., Berkowitz L. and Oneal R.: The spreader flap in primary rhinoplasty. Plast. Reconstr. Surg., 119: 1903, 2007.

12- Gruber R.P., Nahai F., Bogdan M.A. and Friedman G.D.: Changing the convexity and concavity of nasal cartilages and cartilage grafts with horizontal mattress sutures. Part II: Clinical results. Plast. Reconstr. Surg., 115: 595, 2005.

13- Gruber R.P.: Open Rhinoplasty. Clin. Plast. Surg., 15: 95, 1988.

14- Gruber R.P.: Suture correction of nasal tip cartilage concavities. Plast. Reconstr. Surg., 100: 1616, 1997.

15- Fischer H. and Gubisch W.: Nasal valves: Importance and surgical procedures. Facial Plast. Surg., 22: 266, 2006.

16- Gubisch W. and Eichhorn-Sens J.: Overresection of the lower lateral cartilages: A common conceptual mistake with functional and aesthetic consequences. Aesthetic Plast. Surg., 33: 6, 2009.
17- Murakami C.S., Barrera J.E. and Most S.P.: Preserving structural integrity of the alar cartilage in aesthetic rhinoplasty using a cephalic turn-in flap. Arch. Facial Plast. Surg., 11: 126, 2009.

18- Tellioglu A.T. and Cimen K.: Turn-in folding of the cephalic portion of the lateral crus to support the alar rim in rhinoplasty. Aesthetic Plast. Surg., 31: 306, 2007.

19- Gunter J.P. and Friedman R.M.: Lateral crural strut graft: Technique and clinical applications in rhinoplasty. Plast. Reconstr. Surg., 99: 943, 1997.

20- McCollough E.G. and Fedok F.G.: The lateral crural turnover graft: Correction of the concave lateral crus. Laryngoscope, 103: 463, 1993.

21- Gubisch W. and Eichhorn-Sens J.: Overresection of the lower lateral cartilages: A common conceptual mistake with functional and aesthetic consequences. Aesthetic Plast. Surg., 33: 6, 2009.

22- Goksel A. and Vladykina E.: Oblique Turnover Flap for Repositioning and Flattening of the Lateral Crura: A Novel Technique to Manage Cephalic Malposition of Lower Lateral Cartilage. Facial Plast. Surg., 33: 491, 2017. 